\title{
ELUENT CONDITIONS FOR DIRECT INJECTIONS OF INTACT PLASMA SAMPLES ONTO A DUAI MODE COLUMN
}

\section{SHINOBU KUDOH and HIROSHI NAKAMURA*}

Takasaki-Laboratory of SmithKline Beecham Seiyaku K.K., 168 ohyagimachi, Takasaki-shi, Gunma-ken 370, Japan

* Department of Analytical Chemistry, Faculty of Pharmaceutical Sciences, The University of Tokyo, 7-3-1 Hongo, Bunkyo-ku, Tokyo 113, Japan

\begin{abstract}
The use of transmittance at $650 \mathrm{~nm}$ was evaluated to measure plasma protein precipitation. The transmittance reflected the protein precipitation quite well and was useful as a facile method to find out suitable eluent conditions allowing a direct injection of plasma samples onto a dual mode column. By applying the method, precipitation of human plasma and human serum albumin were examined with various conditions. They did not precipitate quickly in water, physiological saline, phosphate/citrate and phosphate buffers of extensive $\mathrm{pH}$ range $(2.32-7.12)$ when incubated at $30^{\circ} \mathrm{C}$. Methanol, acetonitrile, ethanol, isopropanol and acetone did not show any considerable effects on the precipitation when mixed with either saline or the buffers at neutral pHs $(>5)$ up to $30 \%(\mathrm{v} / \mathrm{v})$. At low pHs, however. especially below pH $4,30 \%$ of the organic solvents render the plasma proteins to precipitate in short time of the incubation. On the basis of the above findings, a direct HPLC separation of human plasma spiked with $23 \quad \beta$-lactam antibiotics successfully achieved on a dual mode column, Develosil 60C8-OH-5.
\end{abstract}

Keywords Protein precipitation, plasma samples direct injection, organic solvent protein denaturation, dual mode HPLC column, $\beta$-lactam antibiotics

Since the pioneering reports of protein-coated oDS by Yoshida et al[1] and internal surface reversed-phase (ISRP) packing material by Hagestam and Pinkerton[2], similar types of columns having two mechanisms in their functions have been developed and categorized into 6 design concepts[3]. Their common functions are non-adsorptive size-exclusion of biological macromolecules and dynamic reversed-phase partitioning of small molecules[3] The HPLC columns packed with these unique packing materials have been widely accepted for pretreatment-free drug analyses of biological fluids. However. despite the recommendations[4,5] that the columns should be used with eluents containing not greater than $20 \%$ organic solvents, no detailed information on protein precipitation caused by organic solvents is available. In this study, therefore, we evaluated the use of transmittance at $650 \mathrm{~nm}$ as a facile method to measure precipitation of plasma proteins. The method has been applied to find out suitable eluent conditions for a direct plasma injection on a dual mode HPLC column.

\section{EXPERIMENTAL}

Reagents Human plasma obtained from healthy Japanese male volunteers was pooled and the aliquots were stored at $-70^{\circ} \mathrm{C}$ until required. Human serum albumin [HSA, (A-1653, Sigma Chemical Co. St. Louis, MO, U.S.A.)] was purchased and used by reconstituting it with physiological saline (Fuso Yakuhin Kogyo Co., Osaka, Japan) to give $50 \mathrm{mg} \mathrm{m} \mathrm{m}^{-1}$. Both the plasma and the HSA solution were centrifuged at ca. $10,000 \mathrm{~g}$ for $2 \mathrm{~min}$ and filtered through a $0.22 \mu \mathrm{m}$ membrane filter cartridge (Japan Millipore, Ltd., Tokyo, Japan) just before experiments. Water used throughout this investigation was produced from a Milli-Q SP TOC with a Labo Ionpure 12 apparatus (Japan Millipore). All other chemicals and solvents used were either of analytical or HPLC grade 
(Kanto Chemical Co., Inc. Tokyo, Japan). Isotonic citrate/phosphate buffers at required pHs were prepared by mixing $25 \mathrm{mM}$ citric acid-100mM sodium chloride and $50 \mathrm{mM}$ disodium phosphate $-100 \mathrm{mM}$ sodium chloride in various proportions. In a similar manner, phosphate buffers were also prepared with $80 \mathrm{mM}$ dipotassium phosphate and $160 \mathrm{mM}$ potassium dihydrogenphosphate. Seven types of penicillin (AMPC, ABPC, CBPC, MFIPC, PIPC, PCG and TIPC), 11 types of cephem (CAZ, CER, CEX, CEZ, CFS, CFX, CMX, CMNX, CMZ, CPZ and CTM), 2 types of oxycephem (FMOX and LMOX) a monobactam (AZT) a carbapenem (IPM) and a $\beta$-lactamase inhibitor (CVA) were separately dissolved in the phosphate buffer ( $\mathrm{pH} 7.0)$ and mixed together to give $1 \mu \mathrm{g} \mathrm{ml} \mathrm{m}^{-1}$ for each antibiotics. one hundred micro liter of the mixture was added to $900 \mu 1$ of either saline or the plasma to injected directly onto a dual mode column in HPLC.

Apparatus The HPLC system consisted of a spectra-Physics SP-8800 ternary pump (San Jose, CA, U.S.A.) a Rheodyne Model 7125 injector with a $100 \mu l$ sample loop (Cotati, CA, U.S.A.) and an 0tsuka electronics MCPD-3500 photodiode array UV/VIS detector (Osaka, Japan). A dual mode column used was Develosil 60C8-0H-5 (4.6mmi.d.x 25cm; Nomura Chemical Co., Seto, Japan) and was maintained at $30^{\circ} \mathrm{C}$ by an SSC $3520 \mathrm{C}$ column oven (Senshu Scientific Co. Ltd. Tokyo, Japan). A Hitachi model 220A spectrophotometer with a thermostated cuvette holder (Tokyo, Japan) and Pharmacia Phastsystem electrophoresis (Uppsala, Sweden) were used.

Transmittance measurement Ten micro liter aliquot of the plasma or the HSA solution was added to $500 \mu \mathrm{l}$ of prewarmed $\left(30^{\circ} \mathrm{C}\right)$ appropriate test solution in a plastic vial, gently mixed by inversion for a few times and placed in a water bath at $30^{\circ} \mathrm{C}$ for incubation. At the end of each incubation, the solution was mixed again with a micro-pipette to obtain a homogeneous solution, transferred into a thermostated disposable micro cuvette and the transmittance was measured at $650 \mathrm{~nm}$.

SDS-PAGE The incubated solution was centrifuged at ca.10,000g, and the supernatant was subjected to SDS-PAGE with a PhastGel(type 10-15, Pharmacia) and coomassie staining by following the instruction described in the manual of Pharmacia.

\section{RESULTS and DISCUSSION}

The transmittance of the plasma and the HSA solution were not changed for over $15 \mathrm{~min}$ in water, physiological saline and isotonic phosphate/citrate buffer at $\mathrm{pH}$ ranged 2.32 to 7.12 at $30^{\circ} \mathrm{C}$, indicating that $\mathrm{pH}$ or ion strength has little influence on them. Figure 1 demonstrates a clear response of transmittance changes with increase of methanol content in the isotonic buffer at pH7.12; methanol content over $30 \%$ caused big changes. Also, the

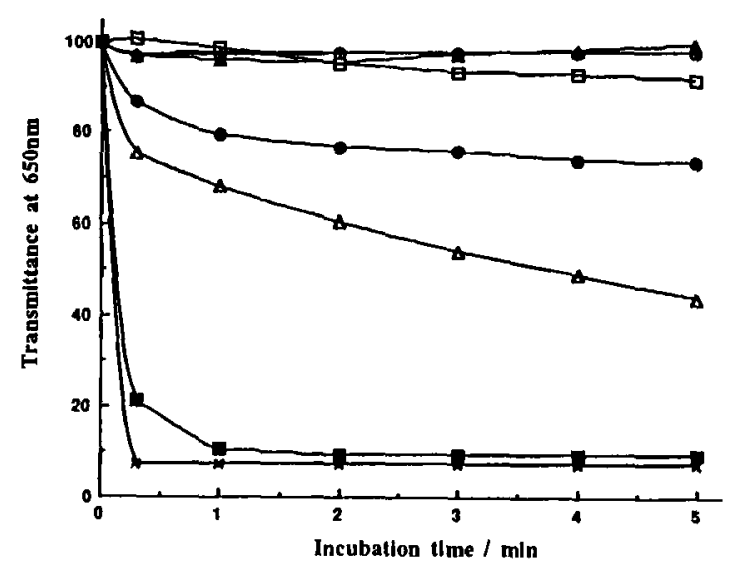

Fig. 1 Transmittance changes of human plasma incubated at $30^{\circ} \mathrm{C}$ in an isotonic phosphate/citrate buffer $(\mathrm{pH}$ $7.12)$ containing varying concentration of methanol. Methanol concentration: + . $0 \% ; 0,10 \% ; \Delta, 20 \% ; \square, 30 \% ; \bigcirc, 40 \%$; $\Delta, 50 \% ; \square, 60 \%$ and $x \quad 70 \%$.

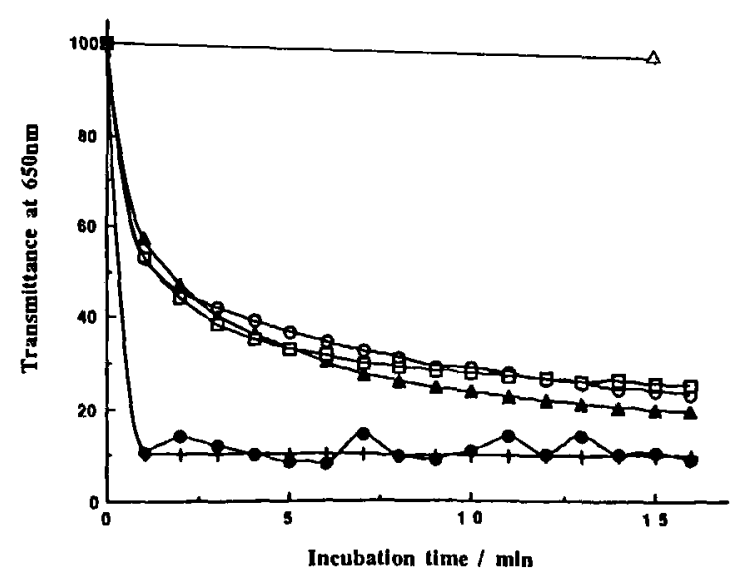

Fig.2 Transmittance changes of human plasma incubated at $30^{\circ} \mathrm{C}$ in saline containing $50 \%$ of organic solvents. Solvent: $\Delta$, water; 0 , methanol; $\Delta$, ethanol: $\square$, isopropanol; $\bullet$, acetonitrile and + , acetone. 
considerable transmittance changes of plasma and the HSA were not observed in saline and the buffer at pH 7.12 containing up to $30 \%$ of acetonitrile, ethanol, isopropanol and acetone as well as methanol. When the contents of the organic solvents were increased as much as $50 \%$, the transmittance rapidly decreased and this was extreme in the case with acetone and acetonitrile as plotted in Fig.2. The electropherograms in Fig.3 well support that these transmittance changes reflected protein precipitation caused by addition of

( I)

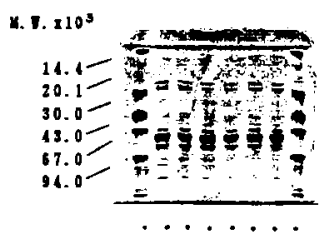

(II)

(III)
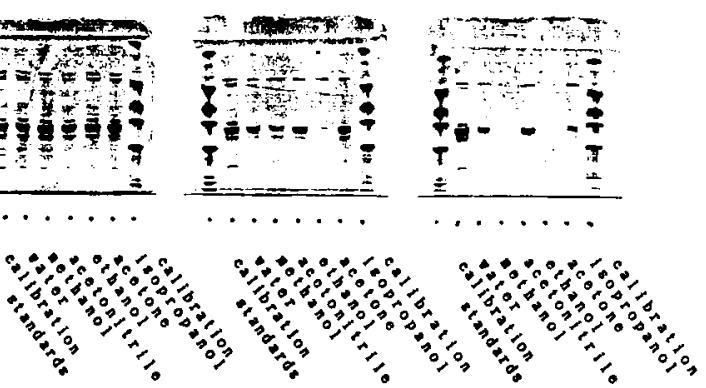

Fig.3 SDS-PAGE of human plasma incubated at $30^{\circ} \mathrm{C}$ in saline containing different organic solvents at (I) $30 \%$, (II) $50 \%$ and (III) $60 \%$. organic solvents. At low pH however, certain collaborative effect of $\mathrm{pH}$ and organic solvent contents on the protein precipitation was observed in the case of methanol (Fig.4) and acetonitrile.

Electropherograms depicted in Fig.5 clearly support the results again that at low $\mathrm{pH}$, especially below pH $4,30 \%$ of either methanol or acetonitrile rendered the plasma protein to precipitate. By applying the information obtained in this study, a direct HPLC separation of human plasma spiked with 23 $\beta$-lactam antibiotics was

successfully achieved on the dual mode Develosil 60C8-0H-5 column (Fig.6). The quantitative recovery of the plasma proteins was spectrophotometrically confirmed.

In conclusion, the transmittance at $650 \mathrm{~nm}$ reflected the protein

precipitation by organic solvents quite well and was useful as a facile method to find out a suitable eluent condition allowing a direct injection of plasma samples onto the dual mode column. By applying this method, informative results could easily be obtained that there would be no problem to use the organic modifiers tested up to $30 \%$ with neutral buffer as an eluent for direct injection. However, great care must be taken to use the organic solvents with acidic buffer for the purpose. By considering elution conditions in this way, a direct HPLC separation of plasma samples can be achieved on the dual mode column. Use of such a type of column as a main analytical column certainly has advantages over use of the column as a precolumn in combination with a reversed-phase analytical column as previously reported [6] when preconcentration is not re-

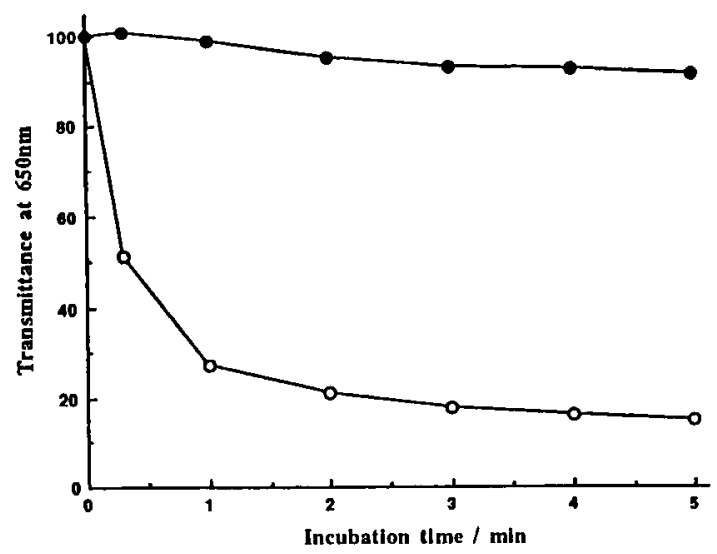
quired.

Fig.4 Transmittance changes of human plasma incubated at $30^{\circ} \mathrm{C}$ at different pH of isotonic phosphate/citrate buffers with $30 \%$ methanol. $\mathrm{pH}: 0,3.04$ and $\bullet, 7.12$.

Fig.5 SDS-PAGE of human plasma incubated at $30^{\circ} \mathrm{C}$ at different $\mathrm{pH}$ of isotonic phosphate/citrate buffers containing $30 \%$ of (I) methanol, (II) acetonitrile and (III) water.

(I)

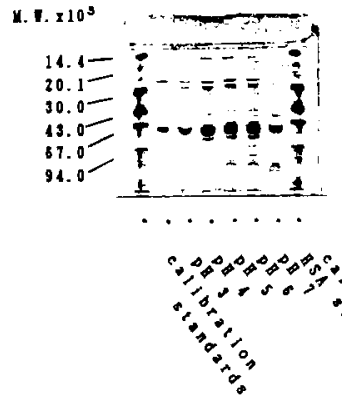

(II)

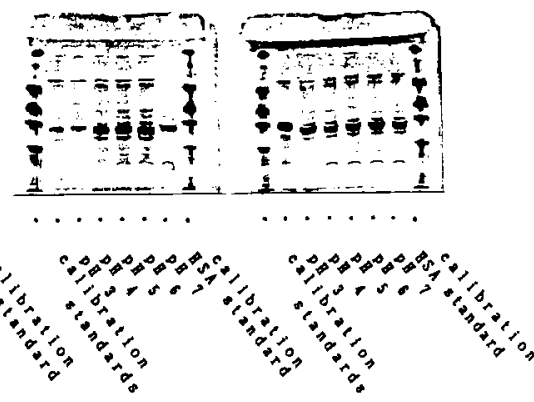


Fig.6 A chromatogram of human plasma spiked with $23 \beta$-lactam antibiotics directly injected. P, Plasma proteins; 1 , CVA; 2 , AMPC; 3 , CMNX; 4, CFS; 5 , LMOX; 6, AZT; 7 CAZ; 8, CTM; 9, TIPC; $10, \mathrm{ABPC} ; 11, \mathrm{CEX} ; 12$, CBPC; 13, FMOX; 14, CEZ; 15 , IPM; $16, \mathrm{CMZ} ; 17, \mathrm{CER} ; 18, \mathrm{CMX}$; 19, CFX; 20, CPZ; 21, PCG; 22, PIPC and 23, MFIPC. Analytical conditions: detection, 230nm; injection volume, $25 \mu 1$; flow rate, $1 \mathrm{ml} \mathrm{min}^{-1}$; elution, a linear gradient of methanol from 0 to $50 \%$ in isotonic phosphate buffer ( $\mathrm{pH} 7.0)$ over 50min.

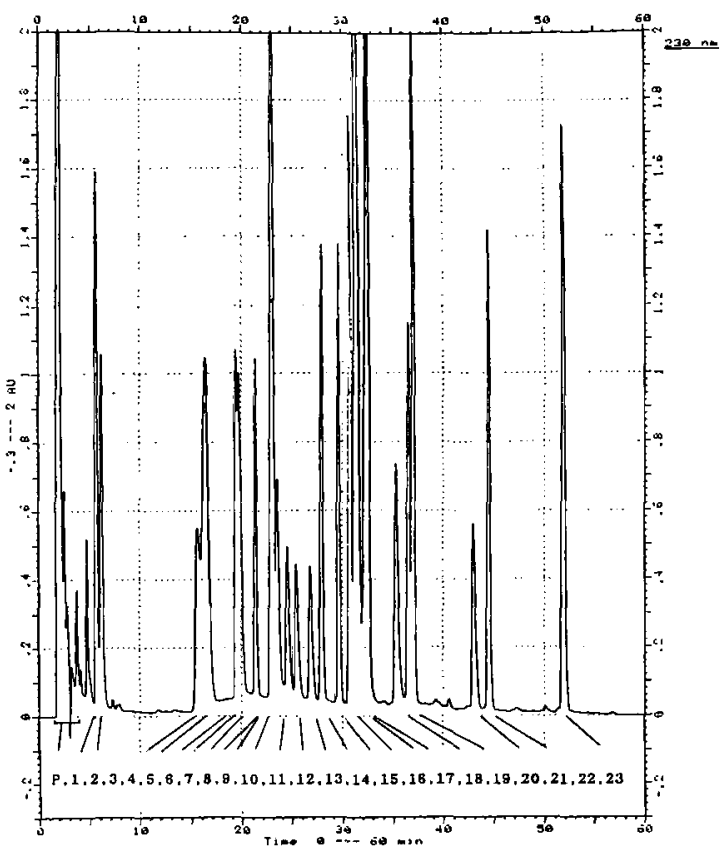

The authors thank Misses Yasue Kurata and Kiyomi Tago for assistance. They also wish to acknowledge the support of Mr. Hiroyuki Kumakura, Dr Noboru Nagahama and Dr. David R. Summers.

\section{REFERENCES}

1. H. Yoshida, T. Morita, T Masuiima and H. Tmai rhom. Pharm. Bull. 30, 3827 (1982)

2. I.H. Hagestam and T.C. Pinkerton, Anal. Chem., 57, 1757 (1985).

3. T.C. Pinkerton, J. Chromatogr $\underline{544}, 13$ (1991).

4. J.A. Perry, J. Liq. Chromatogr. 13,1047 (1990).

5. G.D. George and J.T. Stewart, J. Liq. Chromatogr., 13, 3861 (1990).

6. S. Kudoh and H. Nakamura, Anal. Sci., 5,39 (1989). 